\title{
Epoxide Hydrogenation Catalyzed by Ruthenium PNN and PNP Pincer Complexes
}

\author{
Fallyn L. Kirlin, Olivia J. Borden, Marianna C. Head, Sophie. E. Kelly, and Anthony R. Chianese* \\ Department of Chemistry, Colgate University, 13 Oak Drive, Hamilton, New York 13346, United States
}

\begin{abstract}
The metal-catalyzed hydrogenation of epoxides to give alcohols has advanced rapidly in the past several years, with some catalysts selectively giving linear (anti-Markovnikov) products and other catalysts providing branched (Markovnikov) products. The currently known branched-selective catalyst systems require catalyst loadings of $1 \%$ or higher and typically require a strong base additive. We report herein that PNN- and PNP-ruthenium-pincer complexes containing $\mathrm{N}-\mathrm{H}$ functional groups are highly active for branched-selective hydrogenation of epoxides. When isopropyl alcohol is used as the solvent, excellent yields of the branched alcohol products are obtained without strongly basic additives, using catalyst loadings as low as $0.03 \%$. Epoxides with a directly attached secondary carbon give very high (>99:1) selectivity for the branched products. Aryl-substituted epoxides give branched:linear ratios ranging from 2.7 to 19.0. For aryl epoxides, a PNP-Ru catalyst showed a greater preference for the branched product than a PNN-Ru catalyst, and substrates with electron-rich aryl substituents showed a lower preference for the branched product.
\end{abstract}

\section{Introduction}

The selective hydrogenation and dehydrogenation of polar substrates has advanced steadily since the pioneering work of the Noyori ${ }^{1}$ and Shvo ${ }^{2}$ groups, demonstrating cooperation between a Lewisacidic ruthenium center and a nearby basic site on the ligand. Although excellent reactivity was obtained for ketones and similarly electrophilic substrates, catalysts of this class did not promote the hydrogenation of less reactive carbonyl-containing substrates such as esters and amides. A major advance was achieved when Milstein and co-workers showed that the complex RuPNN ${ }^{\text {dearom }}$ was active for the hydrogenation of esters ${ }^{3}$ and the reverse reaction, the acceptorless dehydrogenative coupling of primary alcohols. ${ }^{4}$ The same PNN-pincer complex was later demonstrated to catalyze the hydrogenation of carbonate esters ${ }^{5}$ and carbon dioxide, ${ }^{6}$ as well as an array of dehydrogenative coupling reactions. ${ }^{7}$ Although the original catalyst RuPNN ${ }^{\text {dearom }}$ lacked an acidic heteroatom site on the ligand (such as $\mathrm{N}-\mathrm{H}$ or $\mathrm{O}-\mathrm{H})$, subsequent catalyst development by many research groups has converged on the beneficial effect of an $\mathrm{N}-\mathrm{H}$ group in close proximity to the metal center for hydrogenation of polar substrates. ${ }^{8}$ Computational studies have shown that this Noyori-type catalyst structure enables the metal-ligandcooperative (MLC) heterolytic splitting of $\mathrm{H}_{2}$ and its transfer to polar substrates with thermally accessible free-energy barriers. ${ }^{8 e, 8 f, 81,8 n, 8 p, 9}$

The originally proposed mechanism for ester hydrogenation catalyzed by RuPNN ${ }^{\text {dearom }}$ involved heterolytic activation of hydrogen by the Lewis-acidic ruthenium center and a basic carbon site on the pincer ligand to give a dihydride intermediate, followed by transfer of these hydrogen atoms to the ester reactant and aldehyde intermediate. ${ }^{3}$ Three computational studies supported the on-cycle intermediacy of this dihydride, albeit with variation in the overall pathway. ${ }^{10}$ However, we recently 
demonstrated experimentally that RuPNN ${ }^{\text {dearom }}$ is not an active catalyst for ester hydrogenation in its own right. ${ }^{11}$ On heating, RuPNN ${ }^{\text {dearom }}$ undergoes a ligand dehydroalkylation reaction to release ethane. In the presence of $\mathrm{PC}_{3}$, the five-coordinate ruthenium(0) species RuPNN ${ }^{\text {imine }}$ is formed, which converts in the presence of hydrogen to the Noyori-type complex RuPNN ${ }^{\text {HEt }}$ (Scheme 1). RuPNN ${ }^{\text {imine }}$ is the most active one-component precatalyst for ester hydrogenation reported to date, giving thousands of turnovers for a range of substates at room temperature with no added base. In a comprehensive experimental and computational study, we demonstrated that RuPNN $\mathbf{N}^{\mathrm{HEt}}$ is a key on-cycle catalytic intermediate, and that the nascent $\mathrm{N}-\mathrm{H}$ group is intimately involved in both hydrogen activation and the transfer of hydrogen to the substrate. ${ }^{12}$

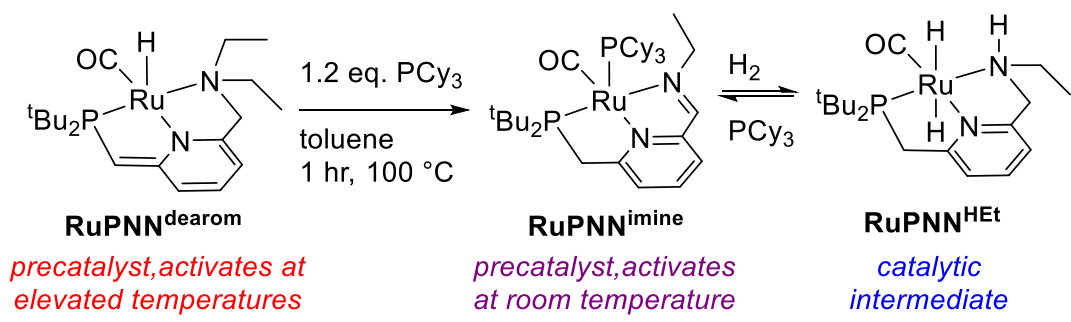

Scheme 1. Conversion of Milstein's Catalyst RuPNN ${ }^{\text {dearom }}$ to the form active for ester hydrogenation.

After demonstrating that RuPNN ${ }^{\text {imine }}$ is a highly active precatalyst for ester hydrogenation, we decided to examine its applicability for the hydrogenation of related polar compounds. In this context, the hydrogenation of epoxides ${ }^{13}$ has been explored less comprehensively. Several catalyst systems have been reported that give the linear (anti-Markovnikov) alcohol as the major product. Norton and coworkers reported a Ti-Co dual catalytic system that selectively gave linear products through a proposed radical mechanism. ${ }^{14}$ Scheuermann and coworkers reported the linear-selective hydrogenation of epoxides using a combination of triflic acid and iridium-PCP-pincer complexes. ${ }^{15}$ In this system, acid catalyzes epoxide hydrolysis to give the 1,2-diol, and iridium nanoparticles formed in situ catalyze diol hydrogenolysis, giving the terminal alcohol as the sole product. ${ }^{16}$ Beller and coworkers described an Fe/tetraphos/trifluoroacetic acid system ${ }^{17}$ and a Co/triphos/Zn(OTf $)_{2}$ system ${ }^{18}$ that selectively gave anti-Markovnikov products. In this work, acid-catalyzed isomerization of the epoxide to the aldehyde was shown to precede transition-metal-catalyzed hydrogenation to give the terminal alcohol. Werner and coworkers reported an erbium-cobalt dual system using ammonia-borane as the hydrogen source, which also gives anti-Markovnikov alcohol products. ${ }^{19}$ In this system, $\operatorname{Er}(\mathrm{OTf})_{3}$ catalyzes initial isomerization to the aldehyde, and a cobalt-pincer complex catalyzes transfer hydrogenation to give the product alcohol. The cobalt systems reported by the Beller ${ }^{18}$ and Werner ${ }^{19}$ groups are also effective for the anti-Markovnikov-selective hydrogenation of 1-aryl-2-alkyl-substituted epoxides and even trisubstituted epoxides.

In contrast to the linear-selective examples described above, some catalysts have been reported to give the branched (Markovnikov) alcohols as major products. Heterogeneous $\mathrm{Pd} / \mathrm{C}$ catalysts typically give a mixture of branched and linear products in the hydrogenation of aliphatic epoxides, ${ }^{13 a}$ although some modified catalysts have shown good selectivity for the branched isomer. ${ }^{20}$ Ikariya and coworkers reported that a $\mathrm{Ru} / \mathrm{Ph}_{2} \mathrm{PCH}_{2} \mathrm{CH}_{2} \mathrm{NH}_{2} / \mathrm{KOH}$ system gave excellent branched selectivity for alkyl-substituted 
epoxides, while the hydrogenation of styrene oxide gave an 89:11 ratio of branched to linear product. ${ }^{21}$ The authors proposed a Noyori-type MLC mechanism for epoxide ring-opening, where $\mathrm{Ru}-\mathrm{H}$ and $\mathrm{N}-\mathrm{H}$ moieties participate in the cooperative transfer of hydrogen to the epoxide substrate. Recently, Thiyagarajan and Gunanathan reported that the commercially available PNP-pincer complex Ru-MACHO in combination with $\mathrm{KO}^{\mathrm{t}} \mathrm{Bu}$ catalyzes the selective hydrogenation of monosubstituted epoxides to the branched products, giving excellent selectivity for alkyl-substituted epoxides and very good selectivity for aryl epoxides. ${ }^{22}$ Again, a Noyori-type MLC mechanism was proposed. Very recently, Jiao, de Vries, Pignataro, and coworkers reported an iron-cyclopentadienone catalyst system that selectively gives the branched or linear product depending on the choice of Lewis-acid cocatalyst. A computational study lent support to a Noyori-type MLC mechanism for both branched and linear products, as well as a competing pathway for linear products involving Lewis-acid-catalyzed isomerization to the aldehyde followed by iron-catalyzed aldehyde hydrogenation. ${ }^{23}$

The reports by Ikariya ${ }^{21}$ and Gunanathan ${ }^{22}$ show that Noyori-type bifunctional ruthenium catalysts have promise in the selective hydrogenation of epoxides, preferentially promoting hydride attack on the less sterically hindered carbon. However, there is room for improvement in catalyst performance. Both reported systems employ catalytic amounts of a strong base - either $\mathrm{KOH}$ or $\mathrm{KO} \mathrm{B}^{\mathrm{B}} \mathrm{u}$ - and both require at least $1 \mathrm{~mol} \%$ of ruthenium catalyst to achieve good yields. Both systems are also limited to monosubstituted epoxides. Although the system reported by Jiao, de Vries, Pignataro, and coworkers ${ }^{23}$ employs the abundant metal iron, it requires a high $5 \%$ catalyst loading and operates at $150{ }^{\circ} \mathrm{C}$. Because RuPNN $^{\text {imine }}$ is a highly active precatalyst for ester hydrogenation and is known to form the Noyori-type catalyst RuPNN ${ }^{\mathrm{HEt}}$ in the presence of hydrogen, we decided to examine its activity for the hydrogenation of epoxides. Additionally, we were curious whether Ru-MACHO-BH, a commercially available borohydride derivative of Ru-MACHO, might catalyze epoxide hydrogenation without the need for added base (Figure 1). ${ }^{24}$ We found that both RuPNN ${ }^{\text {imine }}$ and Ru-MACHO-BH are highly active onecomponent catalysts for branched-selective epoxide hydrogenation, and that the use of isopropyl alcohol as solvent enables high yields to be obtained at catalyst loadings as low as $0.03 \%$.

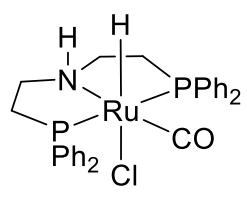

Ru-MACHO

activated by strong base

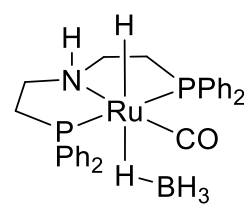

Ru-MACHO-BH

does not require base for activation

Figure 1. Hydridochloride and hydridoborohydride forms of the Ru-MACHO catalyst.

\section{Catalyst Comparison and Optimization}

At the outset of our study, we sought to address three challenges in epoxide hydrogenation: 1) to achieve high yields for a range of substrates with low catalyst loading; 2 ) to eliminate the need to add strong base as a cocatalyst; and 3) to determine whether product racemization can be avoided, potentially enabling the synthesis of chiral secondary alcohols from enantiomerically pure epoxides. We 
chose to compare RuPNN ${ }^{\text {imine }}$, discovered in our laboratory, with Ru-MACHO-BH. ${ }^{24}$ We began by studying the hydrogenation of $(R)$-styrene oxide, monitoring the overall yield, branched:linear (b:I) ratio, and enantiomeric excess (ee) of the branched alcohol product over time. In all cases where an enantioenriched product was formed, the major enantiomer was $(S)$-1-phenylethanol, resulting from retention of configuration at the secondary carbon.

We first compared reactions catalyzed by $1 \mathrm{~mol} \%$ RuPNN ${ }^{\text {imine }}$ or Ru-MACHO-BH at $60{ }^{\circ} \mathrm{C}$ in toluene, with or without $10 \mathrm{~mol} \% \mathrm{NaO}^{t} \mathrm{Bu}$ added (Figure 2). Under these conditions, both ruthenium complexes showed a benefit from added base in terms of the rate of reaction. Reactions catalyzed by Ru-MACHOBH were sluggish, reaching less than $20 \%$ yield after 3 hours even with added base. Interestingly, the b:I ratio increased substantially over time when no base was present, but was nearly constant over time when $\mathrm{NaO}^{\text {t}} \mathrm{Bu}$ was added to the reaction. Early on, reactions catalyzed by Ru-MACHO-BH showed a low but measurable ee, which quickly fell to zero after about two hours of reaction, indicating that the branched alcohol product is racemized under the reaction conditions. Reactions catalyzed by RuPNN ${ }^{\text {imine }}$ showed somewhat faster conversion to product, especially with added base, where an overall yield of $58 \%$ was obtained after 3.5 hours. The addition of base also gave a higher selectivity for the branched product. Completely racemic product was observed at all time points for both experiments with RuPNN ${ }^{\text {imine }}$, indicating that product racemization is much faster than epoxide hydrogenation under these conditions. 

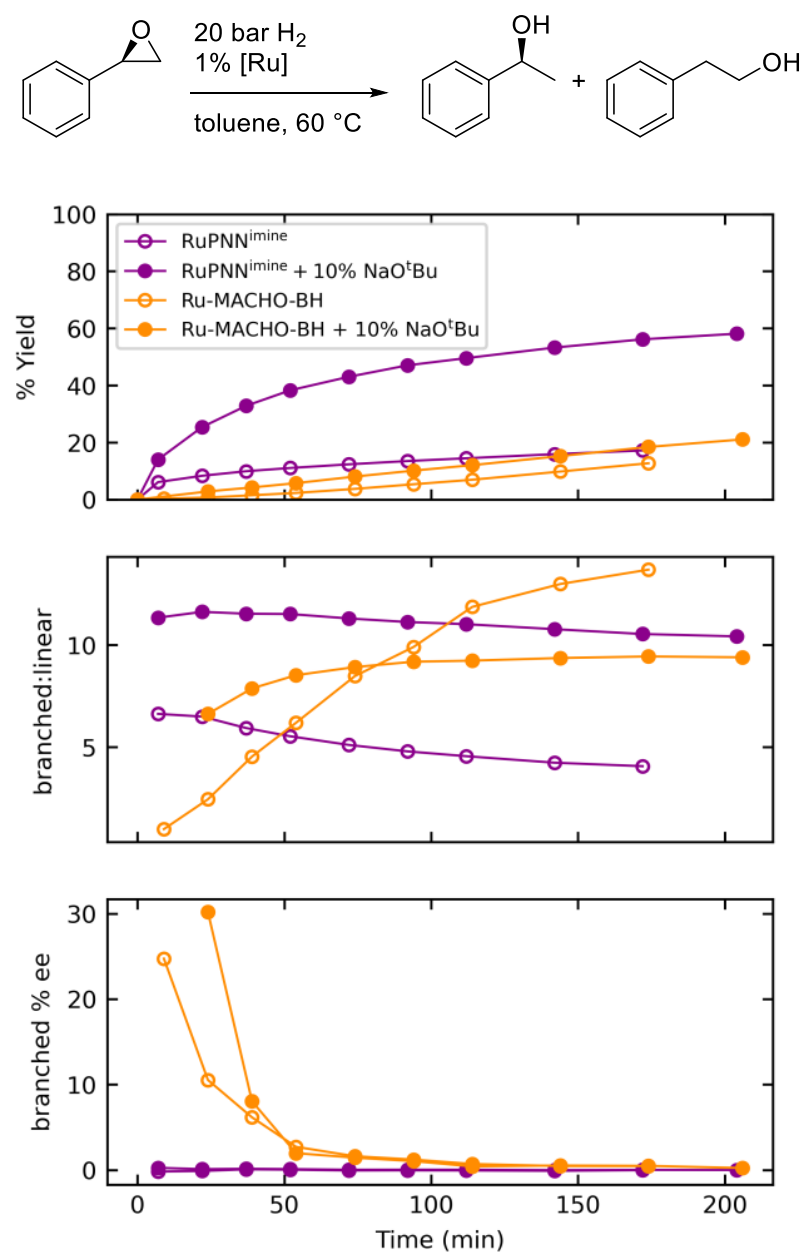

Figure 2. Time-course study of $(R)$-styrene oxide hydrogenation in toluene.

Next, we sought to assess the effect of polar solvents on the hydrogenation of $(R)$-styrene oxide. Although Thiyagarajan and Gunananthan found that a Ru-MACHO/KO'Bu system was effective in toluene, ${ }^{22}$ Ikariya and coworkers reported that isopropyl alcohol was most effective in their $\mathrm{Ru} / \mathrm{Ph}_{2} \mathrm{PCH}_{2} \mathrm{CH}_{2} \mathrm{NH}_{2} / \mathrm{KOH}$ system. ${ }^{21}$ We previously found that isopropyl alcohol was a uniquely effective solvent for ester hydrogenation catalyzed by RuPNN ${ }^{\text {mine }} .{ }^{11}$ Figure 3 shows the time course of the hydrogenation of $(R)$-styrene oxide catalyzed by $1 \mathrm{~mol} \%$ RuPNN ${ }^{\text {imine }}$ at $60^{\circ} \mathrm{C}$ in five polar solvents, with no added base. Very slow conversion was observed in methanol, ethanol, and 1,1,1-trifluoroethanol (TFE). Interestingly, although only $4 \%$ yield was obtained after 3.5 hours in TFE, almost exclusively linear product was formed: the b:I ratio was 1:103 at the end of the reaction. We speculate that this may arise from a combination of catalyst transformation to a less active form and acid-catalyzed isomerization of the epoxide to phenylacetaldehyde in this solvent. Only $23 \%$ yield was reached in THF, although the b:I ratio was very high throughout the reaction, consistently between 17:1 and 19:1. Isopropyl alcohol gave by far the fastest reaction, with the overall yield reaching $90 \%$ after three hours, albeit with lower b:I 
selectivity of 6:1. Complete product racemization was observed for all solvents except ethanol, although only $7 \%$ yield was obtained in ethanol.
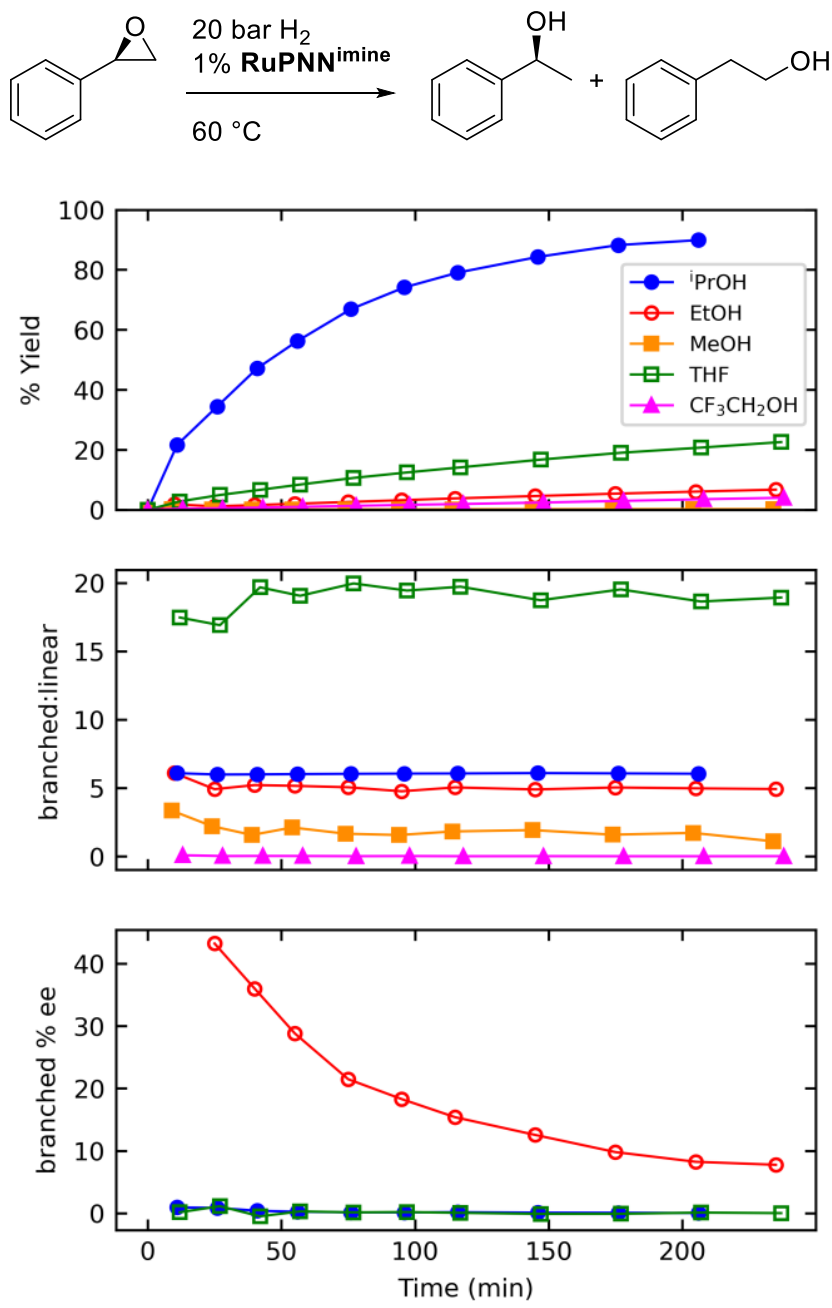

Figure 3. Solvent comparison for $(R)$-styrene oxide hydrogenation catalyzed by RuPNN ${ }^{\text {imine }}$.

We then conducted the same solvent screen using Ru-MACHO-BH as the catalyst, again with no added base (Figure 4). Sluggish conversion was observed in TFE, and again the linear product was predominant: after 3.5 hours, a $5 \%$ overall yield was obtained with a b:I ratio of $1: 30$. The reaction was slow in THF ( $2 \%$ yield) and proceeded at a moderate pace in methanol ( $26 \%$ yield). The imprecision in the measurement of the the b:I over time ratio in THF arises from the very small amount of product formed in this reaction. Encouragingly, a nearly complete reaction was observed in both ethanol ( $94 \%$ yield) and isopropyl alcohol (95\% yield). The highest b:I ratio of 10:1 was seen in isopropyl alcohol. Product racemization was slower with Ru-MACHO-BH than with RuPNN ${ }^{\text {imine }}$, although all solvents other than methanol gave nearly racemic product by the end of the reaction. 

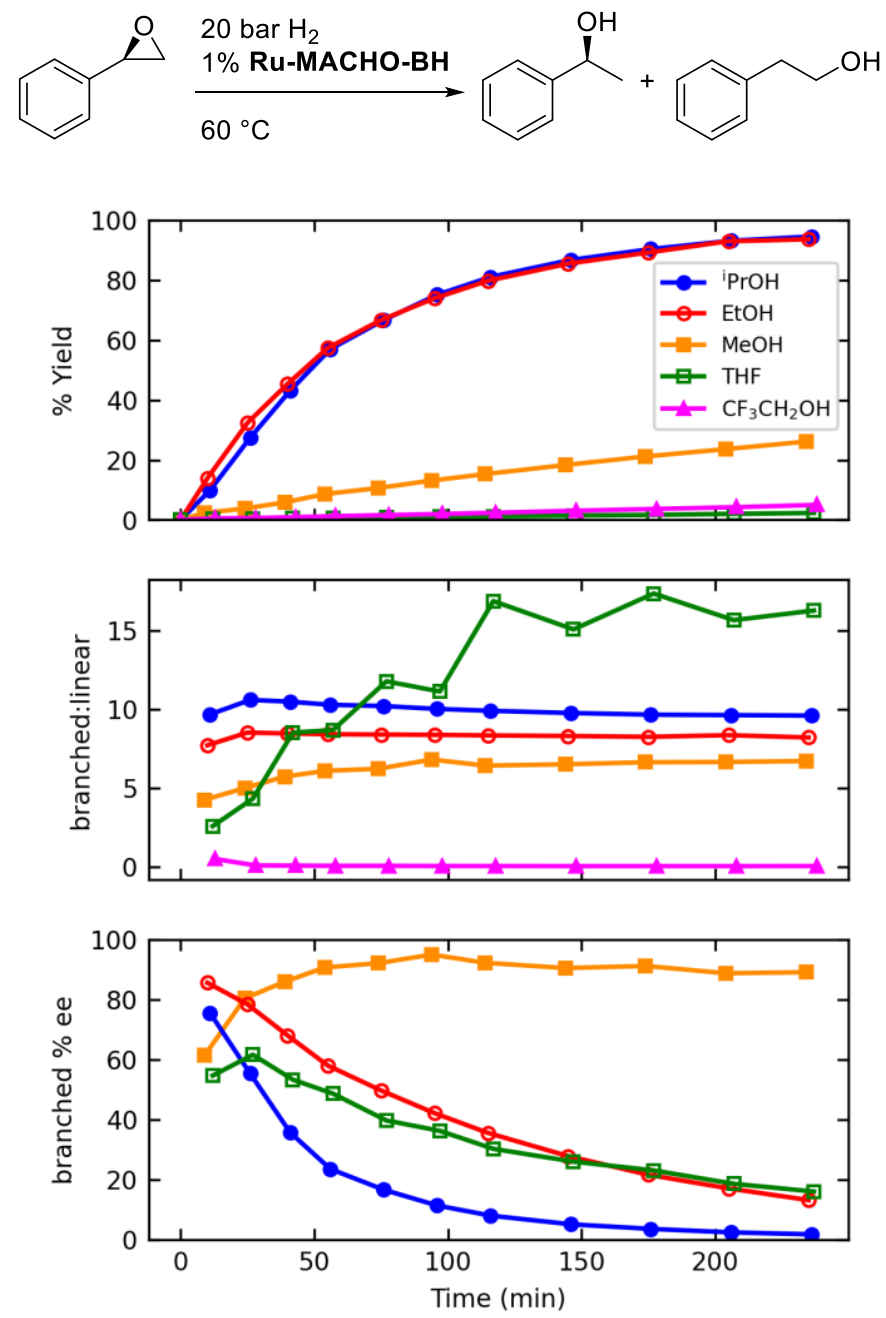

Figure 4. Solvent comparison for (R)-styrene oxide hydrogenation catalyzed by Ru-MACHO-BH.

Although the choice of catalyst and solvent significantly affect the rate of reaction, the b:I ratio, and the product ee, no set of conditions was found that optimizes all three together. For example, very high selectivity for the branched isomer was seen for RuPNN ${ }^{\text {imine }}$ in THF, but a slow overall reaction was observed and the product was completely racemized. On the other hand, minimal product racemization was observed for Ru-MACHO-BH in methanol, but again these conditions gave a slow reaction. Because isopropyl alcohol gave high reaction rates and good b:I ratios with both ruthenium catalysts, we chose this solvent for further study. Plots of the natural logarithm of epoxide concentration vs. time were nearly linear for both experiments conducted in isopropyl alcohol (Figure 5), indicating that the reaction likely displays first-order kinetics with respect to epoxide concentration with both catalysts. 

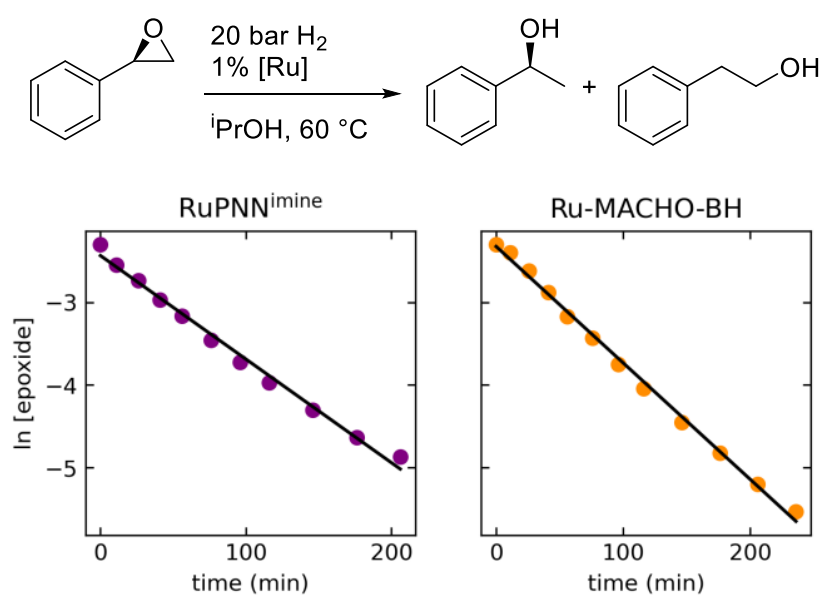

Figure 5. Plots of In[epoxide] vs. time for the hydrogenation of $(R)$-styrene oxide catalyzed by RuPNN ${ }^{\text {imine }}$ and Ru-MACHO-BH.

\section{Phosphine-Catalyzed Solvolysis Side Reaction}

Having identified isopropyl alcohol as an optimal solvent for base-free epoxide hydrogenation catalyzed by RuPNN ${ }^{\text {imine }}$ and Ru-MACHO-BH, we turned our attention to attempting to achieve high yields with low catalyst loadings. The solvent screening experiments described above were conducted with an initial substrate concentration of $0.1 \mathrm{M}$, but the apparent first-order kinetics in epoxide concentration hinted that faster rates could be achieved at higher concentrations. We therefore conducted a series of experiments at a range of catalyst loadings, with $0.5 \mathrm{M} 1$-tetradecene oxide (racemic) in isopropyl alcohol, at a slightly elevated temperature of $80^{\circ} \mathrm{C}$ and an increased hydrogen pressure of 30 bar. The results are collected in Table 1. Under these conditions, Ru-MACHO-BH (Entries 1-4) gave nearly full conversion and an excellent yield of the branched alcohol product $\mathbf{b}$ with a catalyst loading as low as $0.03 \mathrm{~mol} \%$. Surprisingly, the yield of alcohol $\mathbf{b}$ was lower than the conversion with RuPNN $^{\text {imine }}$ as catalyst, and we identified the solvolysis side product s, formed in $5 \%$ yield (Entry 5 ). To investigate the origin of this solvolysis reaction, we performed a series of control experiments. Repeating the reaction with RuPNN ${ }^{\text {imine }}$ as catalyst but without hydrogen gave no hydrogenation product $\mathbf{b}$, but the solvolysis product $\mathbf{s}$ was still formed (Entry 6 ). Interestingly, when $0.5 \%$ RuPNN ${ }^{\text {HEt }}$ was used as catalyst instead, no reaction occurred in the absence of hydrogen and the epoxide substrate was completely recovered (Entry 7 ). When $0.5 \% \mathrm{PCy}_{3}$ was used as catalyst, again without hydrogen, $\mathrm{s}$ was formed as the only observed product in $92 \%$ yield (Entry 8 ). Based on these observations, we concluded that the solvolysis reaction is catalyzed not by the ruthenium complexes in solution, but by the $\mathrm{PCy}_{3}$ released when RuPNN ${ }^{\text {imine }}$ is used as catalyst. To avoid this side reaction, we used RuPNN ${ }^{\text {HEt }}$ directly as the hydrogenation catalyst (Entries 9 and 10). Gratifyingly, the branched alcohol b was formed with excellent yield and selectivity, albeit at a higher loading than with Ru-MACHO-BH (compare Entries 3 and 9). 
Table 1. Solvolysis side reaction catalyzed by $\mathrm{PCy}_{3}$.

\begin{tabular}{|c|c|c|c|c|c|c|}
\hline & $<$ & $\begin{array}{l}0 \text { or } 30 \text { bar } \\
\text { [catalyst] } \\
{ }^{\mathrm{P} r O H} \\
18 \mathrm{~h}, 80^{\circ} \mathrm{C}\end{array}$ & & & $\mathrm{OH}$ & \\
\hline Entry & catalyst & $\mathrm{mol} \%$ & $\mathrm{P}_{\mathrm{H} 2}$ (bar) & $\%$ Conv. & $\%$ Yield $\mathbf{b}$ & $\%$ Yield s \\
\hline 1 & RuMACHO-BH & 0.25 & 30 & 100 & 99 & 0 \\
\hline 2 & RuMACHO-BH & 0.13 & 30 & 100 & 99 & 0 \\
\hline 3 & RuMACHO-BH & 0.063 & 30 & 99 & 98 & 0 \\
\hline 4 & RuMACHO-BH & 0.031 & 30 & 95 & 94 & 0 \\
\hline 5 & RuPNN ${ }^{\text {imine }}$ & 0.13 & 30 & 100 & 95 & 5 \\
\hline 6 & RuPNN ${ }^{\text {imine }}$ & 0.50 & 0 & 13 & 0 & 10 \\
\hline 7 & RuPNN ${ }^{H E t}$ & 0.50 & 0 & 0 & 0 & 0 \\
\hline 8 & $\mathrm{PCy}_{3}$ & 0.50 & 0 & 100 & 0 & 92 \\
\hline 9 & RuPNN ${ }^{H E t}$ & 0.13 & 30 & 99 & 98 & 0 \\
\hline 10 & RuPNN $N^{\text {HEt }}$ & 0.063 & 30 & 77 & 75 & 0 \\
\hline
\end{tabular}




\section{Substrate Scope}

After identifying conditions for selective epoxide hydrogenation with low catalyst loading, we decided to examine the reactivity of a series of epoxide substrates to compare the activity and selectivity of Ru-MACHO-BH vs. RuPNN ${ }^{\text {HEt }}$. For each substrate, we tested a range of catalyst loadings to determine the lowest loading that gave nearly full conversion under the reaction conditions. Table 2 shows the results for monosubstituted epoxides with a directly attached secondary sp ${ }^{3}$-hybridized carbon. For both aliphatic epoxides (Entries 1-4) and glycidyl ethers (Entries 5-8), regioselectivity for the branched alcohol was essentially complete, with less than $1 \%$ of the linear product detected. For each substrate, Ru-MACHO-BH gave full conversion with a lower loading than RuPNN ${ }^{\text {HEt }}$. Substrates in this class also showed high regioselectivity for the branched product in prior work by $1 \mathrm{kariya}^{21}$ and Gunanathan, ${ }^{22}$ although a higher catalyst loading of $1 \%$ or $5 \%$, respectively, was employed in those reports and the addition of catalytic amounts of a strong base was necessary.

Table 2. Hydrogenation of monosubstituted epoxides with an attached secondary carbon.

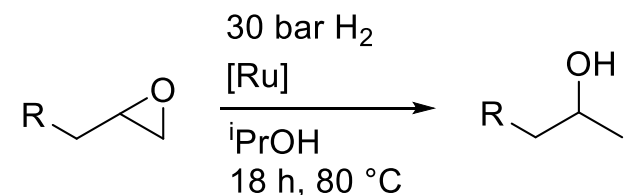

\begin{tabular}{|c|c|c|c|c|c|c|}
\hline Epoxide & Entry & Ru & $\mathrm{mol} \%$ & $\%$ Conv. & $\%$ Yield & $\mathrm{b}: \mathrm{l}$ \\
\hline & 1 & RuMACHO-BH & 0.063 & 99 & 98 & $>99: 1$ \\
\hline & 2 & RuPNN $^{\mathrm{HEt}}$ & 0.13 & 99 & 98 & $>99: 1$ \\
\hline & 3 & RuMACHO-BH & 0.25 & 100 & 97 & $>99: 1$ \\
\hline & 4 & RuPNN ${ }^{\text {HEt }}$ & 0.50 & 100 & 97 & $>99: 1$ \\
\hline & 5 & RuMACHO-BH & 0.063 & 100 & 100 & $>99: 1$ \\
\hline & 6 & RuPNN ${ }^{\mathrm{HEt}}$ & 0.50 & 100 & 96 & $>99: 1$ \\
\hline & 7 & RuMACHO-BH & 0.063 & 99 & 99 & $>99: 1$ \\
\hline & 8 & RuPNN $^{\mathrm{HEt}}$ & 0.13 & 97 & 97 & $>99: 1$ \\
\hline
\end{tabular}


We next examined the reactivity of aryl-substituted epoxides (Table 3), which have shown more variable regioselectivity in previous reports. ${ }^{21-23}$ The parent styrene oxide was converted cleanly to a mixture of the linear and branched phenyl ethanol products with both Ru-MACHO-BH and RuPNN ${ }^{\text {HEt }}$, and Ru-MACHO-BH showed a markedly higher selectivity for the branched product. Substrates with a range of para-substituents were also hydrogenated in high yields. Neither catalyst was effective for substrates with a strongly withdrawing trifluoromethyl or nitro substituent. As was observed for the substrates above in Table 2, Ru-MACHO-BH generally delivered full conversion at a lower loading than RuPNN $^{\text {HEt }}$.

Table 3. Hydrogenation of aryl-substituted epoxides.

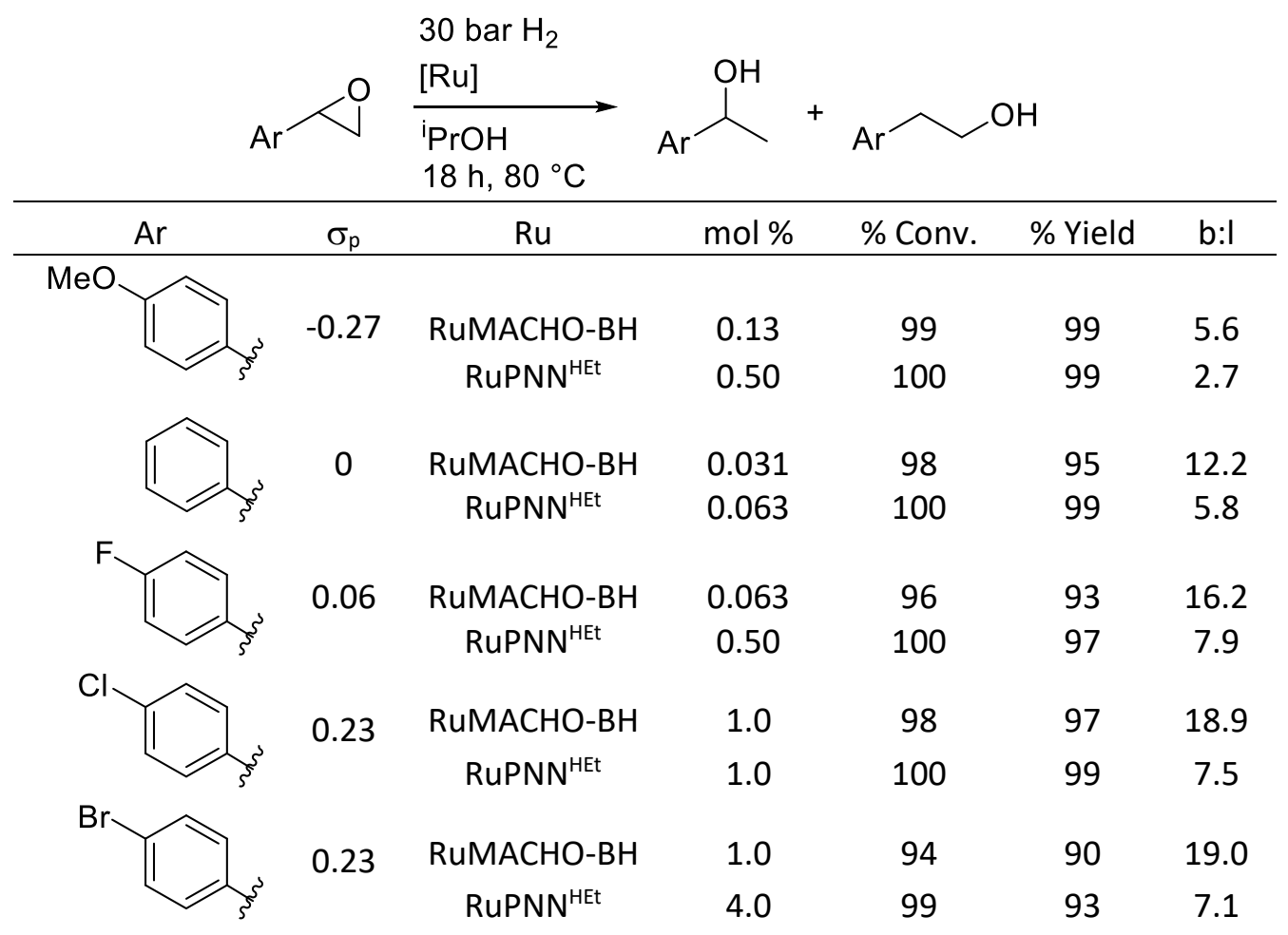

We observed that the branched-to-linear selectivity for aryl epoxides was quite consistent in experiments with different catalyst loadings, and was also consistent over time in individual reactions in isopropyl alcohol (see Figures 3 and 4 above). Therefore, this selectivity is likely kinetic in origin and might be expected to show a linear free-energy relationship with the electronic nature of the parasubstituent. Figure 6 shows Hammett plots ${ }^{25}$ for the five aromatic epoxides that were successfully hydrogenated. For both catalysts, there is a good correlation between the logarithm of the branched:linear ratio and the Hammett constant $\sigma_{\mathrm{p}}$. The positive slope indicates that withdrawing substituents favor the branched product for both RuPNN ${ }^{\text {HEt }}$ and Ru-MACHO-BH. The similar slopes (0.84 for RuPNN ${ }^{\text {HEt }}$ and 1.03 for Ru-MACHO-BH) indicate that the catalysts are similarly sensitive to the parasubstituent, and the greater offset for Ru-MACHO-BH shows that it has a greater intrinsic selectivity for the branched isomer than RuPNN ${ }^{\text {imine }}$ under the reaction conditions employed. A computational and 
experimental mechanistic study on these reactions is currently in progress, and we hesitate to speculate on the mechanistic origin of the substituent effect at this time.

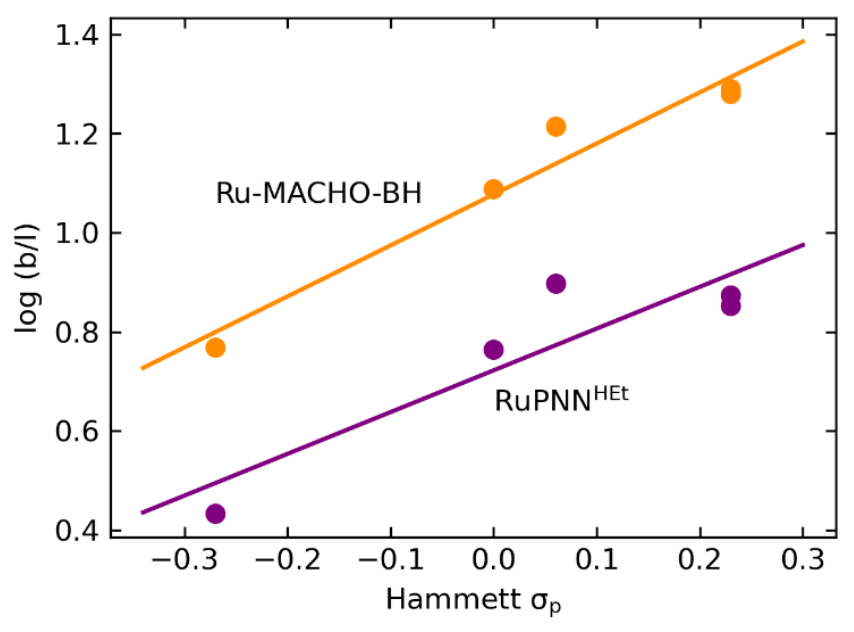

Figure 6. Hammett plots for the b:I selectivity in the hydrogenation of aryl epoxides. For Ru-MACHO-BH, the linear regression equation is $y=1.03 x+1.08$, with $R^{2}=0.96$. For RuPNN ${ }^{\mathrm{HEt}}$, the equation is $y=0.84 x$ +0.72 , with $\mathrm{R}^{2}=0.82$.

Finally, we were interested to assess the activity of RuPNN ${ }^{\text {HEt }}$ and Ru-MACHO-BH for the hydrogenation of disubstituted epoxides. Although the linear-selective catalyst systems described in the introduction are generally effective for the hydrogenation of disubstituted epoxides, ${ }^{14-15,17-19}$ the Noyoritype ruthenium catalysts reported previously are not. Ikariya's catalyst system employing $\mathrm{PPh}_{2} \mathrm{CH}_{2} \mathrm{CH}_{2} \mathrm{NH}_{2}$ as ligand was ineffective for epoxides with more than one substituent, ${ }^{21}$ and Gunanathan's Ru-MACHO/KO'Bu system showed no conversion of stilbene oxide and gave approximately a 1:1 ratio of branched and linear alcohols from 2-phenylpropylene oxide with $5 \%$ catalyst loading. ${ }^{22}$ Table 4 shows the results for three disubstituted epoxides using RuPNN ${ }^{\text {HEt }}$ or RuMACHO-BH in isopropyl alcohol with no added base. Trans-stilbene oxide (racemic) is hydrogenated fully by both catalysts, although a higher $2 \%$ catalyst loading is required. Interestingly, this substrate was reported to be completely unreactive under the conditions reported by Gunanathan (Ru-MACHO/KOt Bu in toluene at $\left.75^{\circ} \mathrm{C}\right) .{ }^{22}$ Cyclohexene oxide is also hydrogenated by both catalysts, although full conversion was not obtained with either catalyst even at a very high $4 \%$ catalyst loading. The 1,1-disubstituted substrate 2-phenylpropylene oxide was not hydrogenated effectively with Ru-MACHO-BH under the standard conditions, but RuPNN ${ }^{\text {HEt }}$ gave full conversion and $99 \%$ yield when a $4 \%$ catalyst loading was used. We speculated that the lack of reactivity of Ru-MACHO-BH might be partially due to the catalyst's low solubility in isopropyl alcohol. Gratifyingly, a 95\% yield was obtained when a 1:1 mixture of toluene and isopropyl alcohol was used as the solvent. With both catalysts, only the branched product was detected for this substrate. To our knowledge, this is the first report of Markovnikov-selective hydrogenation of this commercially available epoxide. 
Table 4. Hydrogenation of disubstituted epoxides.

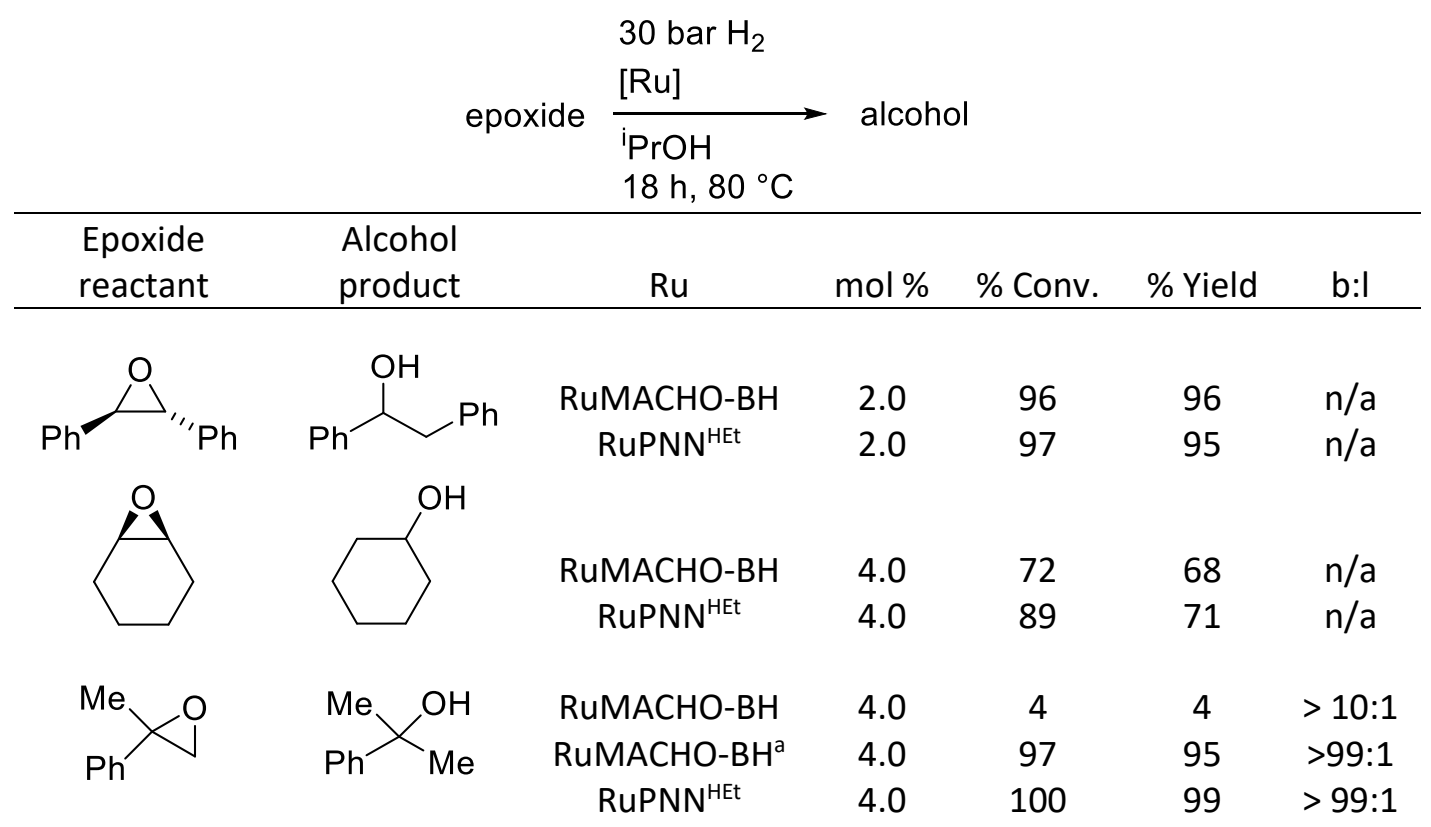

${ }^{a}$ A 1:1 mixture of toluene and isopropyl alcohol was used as solvent.

\section{Summary and Conclusion}

We have shown that RuPNN ${ }^{\text {HEt }}$ and Ru-MACHO-BH are highly effective catalysts for the branchedselective hydrogenation of epoxides without the requirement of strongly basic additives. The use of isopropyl alcohol as solvent enables the complete conversion of epoxides to alcohols in high yield with low catalyst loadings. From a practical standpoint, the use of Ru-MACHO-BH is recommended for most applications due to its commercial availability and moderate cost (\$95/g from Strem). In contrast, RuPNN $^{\text {HEt }}$ is synthesized in two steps ${ }^{12}$ from the commercially available Milstein Catalyst Precursor (\$788/g from Strem). In our experiments, Ru-MACHO-BH also typically gave full substrate conversion at lower loadings compared to RuPNN ${ }^{\mathrm{HEt}}$. An experimental and computational mechanistic study of these reactions is currently in progress.

\section{Author Information}

\section{Corresponding Author}

*achianese@colgate.edu

\section{ORCID}

Fallyn L. Kirlin: 0000-0003-1180-471X

Olivia J. Borden: 0000-0002-5567-3566

Marianna C. Head: 0000-0002-7158-6630

Sophie. E. Kelly: 0000-0001-8350-3671

Anthony R. Chianese: 0000-0002-9140-6115 


\section{Supporting Information}

Experimental procedures for epoxide hydrogenation and numerical data presented in Figures 2-4.

\section{Conflicts of interest}

The authors declare no competing financial interest.

\section{Acknowledgements}

We thank the National Science Foundation (CHE-1954924) for support of this research.

\section{References}

(1) Noyori, R.; Ohkuma, T. Asymmetric Catalysis by Architectural and Functional Molecular Engineering: Practical Chemo- and Stereoselective Hydrogenation of Ketones. Angew. Chem. Int. Ed. 2001, 40, 40-73.

(2) Blum, Y.; Czarkie, D.; Rahamim, Y.; Shvo, Y. (Cyclopentadienone)Ruthenium Carbonyl Complexes - a New Class of Homogeneous Hydrogenation Catalysts. Organometallics 1985, 4, 1459-1461.

(3) Zhang, J.; Leitus, G.; Ben-David, Y.; Milstein, D. Efficient Homogeneous Catalytic Hydrogenation of Esters to Alcohols. Angew. Chem. Int. Ed. 2006, 45, 1113-1115.

(4) Zhang, J.; Leitus, G.; Ben-David, Y.; Milstein, D. Facile Conversion of Alcohols into Esters and Dihydrogen Catalyzed by New Ruthenium Complexes. J. Am. Chem. Soc. 2005, 127, $10840-$ 10841.

(5) Balaraman, E.; Gunanathan, C.; Zhang, J.; Shimon, L. J. W.; Milstein, D. Efficient Hydrogenation of Organic Carbonates, Carbamates and Formates Indicates Alternative Routes to Methanol Based on $\mathrm{CO}_{2}$ and CO. Nature Chem. 2011, 3, 609-614.

(6) Huff, C. A.; Sanford, M. S. Catalytic $\mathrm{CO}_{2}$ Hydrogenation to Formate by a Ruthenium Pincer Complex. ACS Catal. 2013, 3, 2412-2416.

(7) (a) Gunanathan, C.; Ben-David, Y.; Milstein, D. Direct Synthesis of Amides from Alcohols and Amines with Liberation of $\mathrm{H}_{2}$. Science 2007, 317, 790-792. (b) Gnanaprakasam, B.; Balaraman, E.; BenDavid, Y.; Milstein, D. Synthesis of Peptides and Pyrazines from Beta-Amino Alcohols through Extrusion of $\mathrm{H}_{2}$ Catalyzed by Ruthenium Pincer Complexes: Ligand-Controlled Selectivity. Angew. Chem. Int. Ed. 2011, 50, 12240-12244. (c) Gnanaprakasam, B.; Milstein, D. Synthesis of Amides from Esters and Amines with Liberation of $\mathrm{H}_{2}$ under Neutral Conditions. J. Am. Chem. Soc. 2011, 133, 1682-1685. (d) Zeng, H.; Guan, Z. Direct Synthesis of Polyamides Via Catalytic Dehydrogenation of Diols and Diamines. J. Am. Chem. Soc. 2011, 133, 1159-1161. (e) Rigoli, J. W.; Moyer, S. A.; Pearce, S. D.; Schomaker, J. M. Alpha,Beta-Unsaturated Imines Via RuCatalyzed Coupling of Allylic Alcohols and Amines. Org. Biomol. Chem. 2012, 10, 1746-1749.

(8) (a) Saudan, L. A.; Saudan, C. M.; Debieux, C.; Wyss, P. Dihydrogen Reduction of Carboxylic Esters to Alcohols under the Catalysis of Homogeneous Ruthenium Complexes: High Efficiency and Unprecedented Chemoselectivity. Angew. Chem. Int. Ed. 2007, 46, 7473-7476. (b) Takebayashi, S.; Bergens, S. H. Facile Bifunctional Addition of Lactones and Esters at Low Temperatures. The 
First Intermediates in Lactone/Ester Hydrogenations. Organometallics 2009, 28, 2349-2351. (c) Spasyuk, D.; Gusev, D. G. Acceptorless Dehydrogenative Coupling of Ethanol and Hydrogenation of Esters and Imines. Organometallics 2012, 31, 5239-5242. (d) Carpenter, I.; Eckelmann, S. C.; Kuntz, M. T.; Fuentes, J. A.; France, M. B.; Clarke, M. L. Convenient and Improved Protocols for the Hydrogenation of Esters Using Ru Catalysts Derived from $(P, P),(P, N, N)$ and $(P, N, O)$ Ligands. Dalton Trans. 2012, 41, 10136-10140. (e) O, W. W. N.; Morris, R. H. Ester Hydrogenation Catalyzed by a Ruthenium(II) Complex Bearing an N-Heterocyclic Carbene Tethered with an " $\mathrm{NH}_{2}$ " Group and a DFT Study of the Proposed Bifunctional Mechanism. ACS Catal. 2013, 3, 3240. (f) Spasyuk, D.; Smith, S.; Gusev, D. G. Replacing Phosphorus with Sulfur for the Efficient Hydrogenation of Esters. Angew. Chem. Int. Ed. 2013, 52, 2538-2542. (g) Li, W.; Xie, J.-H.; Yuan, M.-L.; Zhou, Q.-L. Ruthenium Complexes of Tetradentate Bipyridine Ligands: Highly Efficient Catalysts for the Hydrogenation of Carboxylic Esters and Lactones. Green Chem. 2014, 16, $4081-$ 4085. (h) Fogler, E.; Garg, J. A.; Hu, P.; Leitus, G.; Shimon, L. J. W.; Milstein, D. System with Potential Dual Modes of Metal-Ligand Cooperation: Highly Catalytically Active Pyridine-Based PNNH-Ru Pincer Complexes. Chem. Eur. J. 2014, 20, 15727-15731. (i) Hu, P.; Fogler, E.; DiskinPosner, Y.; Iron, M. A.; Milstein, D. A Novel Liquid Organic Hydrogen Carrier System Based on Catalytic Peptide Formation and Hydrogenation. Nature Commun. 2015, 6, 6859-6865. (j) Fuentes, J. A.; Smith, S. M.; Scharbert, M. T.; Carpenter, I.; Cordes, D. B.; Slawin, A. M. Z.; Clarke, M. L. On the Functional Group Tolerance of Ester Hydrogenation and Polyester Depolymerisation Catalysed by Ruthenium Complexes of Tridentate Aminophosphine Ligands. Chem. Eur. J. 2015, 21, 10851-10860. (k) Filonenko, G. A.; Aguila, M. J. B.; Schulpen, E. N.; van Putten, R.; Wiecko, J.; Müller, C.; Lefort, L.; Hensen, E. J. M.; Pidko, E. A. Bis-N-Heterocyclic Carbene Aminopincer Ligands Enable High Activity in Ru-Catalyzed Ester Hydrogenation. J. Am. Chem. Soc. 2015, 137, 7620-7623. (I) Spasyuk, D.; Vicent, C.; Gusev, D. G. Chemoselective Hydrogenation of Carbonyl Compounds and Acceptorless Dehydrogenative Coupling of Alcohols. J. Am. Chem. Soc. 2015, 137, 3743-3746. (m) Tan, X.; Wang, Q.; Liu, Y.; Wang, F.; Lv, H.; Zhang, X. A New Designed Hydrazine Group-Containing Ruthenium Complex Used for Catalytic Hydrogenation of Esters. Chem. Commun. 2015, 51, 12193-12196. (n) Tan, X.; Wang, Y.; Liu, Y.; Wang, F.; Shi, L.; Lee, K. H.; Lin, Z.; Lv, H.; Zhang, X. Highly Efficient Tetradentate Ruthenium Catalyst for Ester Reduction: Especially for Hydrogenation of Fatty Acid Esters. Org. Lett. 2015, 17, 454-457. (o) Ogata, O.; Nakayama, Y.; Nara, H.; Fujiwhara, M.; Kayaki, Y. Atmospheric Hydrogenation of Esters Catalyzed by PNP-Ruthenium Complexes with an N-Heterocyclic Carbene Ligand. Org. Lett. 2016, 18, 3894-3897. (p) Wang, Z.; Chen, X.; Liu, B.; Liu, Q.-B.; Solan, G. A.; Yang, X.; Sun, W.-H. Cooperative Interplay between a Flexible PNN-Ru(II) Complex and a $\mathrm{Nabh}_{4}$ Additive in the Efficient Catalytic Hydrogenation of Esters. Catal. Sci. Technol. 2017, 7, 1297-1304. (q) Espinosa-Jalapa, N. A.; Nerush, A.; Shimon, L. J. W.; Leitus, G.; Avram, L.; BenDavid, Y.; Milstein, D. Manganese-Catalyzed Hydrogenation of Esters to Alcohols. Chem. Eur. J. 2017, 23, 5934-5938. (r) Kumar, A.; Janes, T.; Espinosa-Jalapa, N. A.; Milstein, D. Selective Hydrogenation of Cyclic Imides to Diols and Amines and Its Application in the Development of a Liquid Organic Hydrogen Carrier. J. Am. Chem. Soc. 2018, 140, 7453-7457. (s) Kumar, A.; Janes, T.; Espinosa-Jalapa, N. A.; Milstein, D. Manganese Catalyzed Hydrogenation of Organic Carbonates to Methanol and Alcohols. Angew. Chem. Int. Ed. 2018, 57, 12076-12080. (t) Xie, Y.; Hu, P.; Ben-David, Y.; Milstein, D. A Reversible Liquid Organic Hydrogen Carrier System Based on Methanol-Ethylenediamine and Ethylene Urea. Angew. Chem. Int. Ed. 2019, 58, 5105-5109.

(9) (a) Otsuka, T.; Ishii, A.; Dub, P. A.; Ikariya, T. Practical Selective Hydrogenation of Alpha-Fluorinated Esters with Bifunctional Pincer-Type Ruthenium(II) Catalysts Leading to Fluorinated Alcohols or Fluoral Hemiacetals. J. Am. Chem. Soc. 2013, 135, 9600-9603. (b) Chakraborty, S.; Lagaditis, P. O.; Förster, M.; Bielinski, E. A.; Hazari, N.; Holthausen, M. C.; Jones, W. D.; Schneider, S. Well- 
Defined Iron Catalysts for the Acceptorless Reversible Dehydrogenation-Hydrogenation of Alcohols and Ketones. ACS Catal. 2014, 4, 3994-4003. (c) Chen, T.; Li, H.; Qu, S.; Zheng, B.; He, L.; Lai, Z.; Wang, Z.-X.; Huang, K.-W. Hydrogenation of Esters Catalyzed by Ruthenium PN $\mathrm{PN}_{3}$-Pincer Complexes Containing an Aminophosphine Arm. Organometallics 2014, 33, 4152-4155. (d) Junge, K.; Wendt, B.; Jiao, H.; Beller, M. Iridium-Catalyzed Hydrogenation of Carboxylic Acid Esters. ChemCatChem 2014, 6, 2810-2814. (e) Qu, S.; Dai, H.; Dang, Y.; Song, C.; Wang, Z.-X.; Guan, H. Computational Mechanistic Study of Fe-Catalyzed Hydrogenation of Esters to Alcohols: Improving Catalysis by Accelerating Precatalyst Activation with a Lewis Base. ACS Catal. 2014, 4, 4377-4388. (f) Werkmeister, S.; Junge, K.; Wendt, B.; Alberico, E.; Jiao, H.; Baumann, W.; Junge, H.; Gallou, F.; Beller, M. Hydrogenation of Esters to Alcohols with a Well-Defined Iron Complex. Angew. Chem. Int. Ed. 2014, 53, 8722-8726. (g) Chen, X.; Jing, Y.; Yang, X. Unexpected Direct Hydride Transfer Mechanism for the Hydrogenation of Ethyl Acetate to Ethanol Catalyzed by SNS Pincer Ruthenium Complexes. Chem. Eur. J. 2016, 22, 1950-1957. (h) Gusev, D. G. Dehydrogenative Coupling of Ethanol and Ester Hydrogenation Catalyzed by Pincer-Type YNP Complexes. ACS Catal. 2016, 6, 6967-6981. (i) van Putten, R.; Uslamin, E. A.; Garbe, M.; Liu, C.; Gonzalez-de-Castro, A.; Lutz, M.; Junge, K.; Hensen, E. J. M.; Beller, M.; Lefort, L.; Pidko, E. A. Non-Pincer-Type Manganese Complexes as Efficient Catalysts for the Hydrogenation of Esters. Angew. Chem. Int. Ed. 2017, 56, 7531-7534.

(10) (a) Li, H.; Wang, X.; Huang, F.; Lu, G.; Jiang, J.; Wang, Z.-X. Computational Study on the Catalytic Role of Pincer Ruthenium(II)-PNN Complex in Directly Synthesizing Amide from Alcohol and Amine: The Origin of Selectivity of Amide over Ester and Imine. Organometallics 2011, 30, 5233-5247. (b) Wang, H.; Liu, C.; Zhang, D. Decisive Effects of Solvent and Substituent on the Reactivity of Ru-Catalyzed Hydrogenation of Ethyl Benzoate to Benzyl Alcohol and Ethanol: A DFT Study. Mol. Catal. 2017, 440, 120-132. (c) Gusev, D. G. Revised Mechanisms of the Catalytic Alcohol Dehydrogenation and Ester Reduction with the Milstein PNN Complex of Ruthenium. Organometallics 2020, 39, 258-270.

(11) He, T.; Buttner, J. C.; Reynolds, E. F.; Pham, J.; Malek, J. C.; Keith, J. M.; Chianese, A. R. Dehydroalkylative Activation of CNN- and PNN-Pincer Ruthenium Catalysts for Ester Hydrogenation. J. Am. Chem. Soc. 2019, 141, 17404-17413.

(12) Pham, J.; Jarczyk, C. E.; Reynolds, E. F.; Kelly, S. E.; Kim, T.; He, T.; Keith, J. M.; Chianese, A. R. The Key Role of the Latent N-H Group in Milstein's Catalyst for Ester Hydrogenation. Chem. Sci. 2021, 12, 8477-8492.

(13) (a) Thiery, E.; Le Bras, J.; Muzart, J. Reactivity Versus Stability of Oxiranes under Palladium-Catalyzed Reductive Conditions. Eur. J. Org. Chem. 2009, 2009, 961-985. (b) Huang, C. Y.; Doyle, A. G. The Chemistry of Transition Metals with Three-Membered Ring Heterocycles. Chem. Rev. 2014, 114, 8153-98.

(14) Yao, C.; Dahmen, T.; Gansäuer, A.; Norton, J. Anti-Markovnikov Alcohols Via Epoxide Hydrogenation through Cooperative Catalysis. Science 2019, 364, 764-767.

(15) Rainsberry, A. N.; Sage, J. G.; Scheuermann, M. L. Iridium-Promoted Conversion of Terminal Epoxides to Primary Alcohols under Acidic Conditions Using Hydrogen. Catal. Sci. Technol. 2019, 9, 3020-3022.

(16) Gitnes, R. M.; Wang, M.; Bao, Y.; Scheuermann, M. L. In Situ Generation of Catalytically Relevant Nanoparticles from a Molecular Pincer Iridium Precatalyst During Polyol Deoxygenation. ACS Catal. 2021, 11, 495-501.

(17) Liu, W.; Li, W.; Spannenberg, A.; Junge, K.; Beller, M. Iron-Catalysed Regioselective Hydrogenation of Terminal Epoxides to Alcohols under Mild Conditions. Nature Catalysis 2019, 2, 523-528.

(18) Liu, W.; Leischner, T.; Li, W.; Junge, K.; Beller, M. A General Regioselective Synthesis of Alcohols by Cobalt-Catalyzed Hydrogenation of Epoxides. Angew. Chem. Int. Ed. 2020, 59, 11321-11324. 
(19) Liu, X.; Longwitz, L.; Spiegelberg, B.; Tönjes, J.; Beweries, T.; Werner, T. Erbium-Catalyzed Regioselective Isomerization-Cobalt-Catalyzed Transfer Hydrogenation Sequence for the Synthesis of Anti-Markovnikov Alcohols from Epoxides under Mild Conditions. ACS Catal. 2020, 10, 13659-13667.

(20) (a) Sajiki, H.; Hattori, K.; Hirota, K. Pd/C(En)-Catalyzed Regioselective Hydrogenolysis of Terminal Epoxides to Secondary Alcohols. Chem. Commun. 1999, 1041-1042. (b) Kwon, M. S.; Park, I. S.; Jang, J. S.; Lee, J. S.; Park, J. Magnetically Separable Pd Catalyst for Highly Selective Epoxide Hydrogenolysis under Mild Conditions. Org. Lett. 2007, 9, 3417-3419.

(21) Ito, M.; Hirakawa, M.; Osaku, A.; Ikariya, T. Highly Efficient Chemoselective Hydrogenolysis of Epoxides Catalyzed by a (H5-C5(CH3)5)Ru Complex Bearing a 2-(Diphenylphosphino)Ethylamine Ligand. Organometallics 2003, 22, 4190-4192.

(22) Thiyagarajan, S.; Gunanathan, C. Ruthenium-Catalyzed Selective Hydrogenation of Epoxides to Secondary Alcohols. Org. Lett. 2019, 21, 9774-9778.

(23) Tadiello, L.; Gandini, T.; Stadler, B. M.; Tin, S.; Jiao, H.; de Vries, J. G.; Pignataro, L.; Gennari, C. Regiodivergent Reductive Opening of Epoxides by Catalytic Hydrogenation Promoted by a (Cyclopentadienone)Iron Complex. ACS Catal. 2021, 12, 235-246.

(24) (a) Monney, A.; Barsch, E.; Sponholz, P.; Junge, H.; Ludwig, R.; Beller, M. Base-Free Hydrogen Generation from Methanol Using a Bi-Catalytic System. Chem. Commun. 2014, 50, 707-709. (b) Fairweather, N. T.; Gibson, M. S.; Guan, H. Homogeneous Hydrogenation of Fatty Acid Methyl Esters and Natural Oils under Neat Conditions. Organometallics 2015, 34, 335-339. (c) Neumann, J.; Bornschein, C.; Jiao, H.; Junge, K.; Beller, M. Hydrogenation of Aliphatic and Aromatic Nitriles Using a Defined Ruthenium PNP Pincer Catalyst. Eur. J. Org. Chem. 2015, 2015, 5944-5948. (d) Kothandaraman, J.; Goeppert, A.; Czaun, M.; Olah, G. A.; Prakash, G. K. Conversion of $\mathrm{CO} 2$ from Air into Methanol Using a Polyamine and a Homogeneous Ruthenium Catalyst. J. Am. Chem. Soc. 2016, 138, 778-81.

(25) Hansch, C.; Leo, A.; Taft, R. W. A Survey of Hammett Substituent Constants and Resonance and Field Parameters. Chem. Rev. 2002, 91, 165-195. 